IMA Journal of Numerical Analysis (2005) Page 1 of 19

doi: 10.1093/imanum/dri017

\title{
Flexible Penalty Functions for Nonlinear Constrained Optimization
}

\author{
FRANK E. CURTIS * \\ Department of Industrial Engineering and Management Sciences \\ Northwestern University, Evanston, IL, 60208-3118, USA. \\ JORGE NOCEDAL $†$ \\ Department of Electrical Engineering and Computer Science \\ Northwestern University, Evanston, IL, 60208-3118, USA.
}

[Received on 31 March 2007]

\begin{abstract}
We propose a globalization strategy for nonlinear constrained optimization. The method employs a "flexible" penalty function to promote convergence, where during each iteration the penalty parameter can be chosen as any number within a prescribed interval, rather than a fixed value. This increased flexibility in the step acceptance procedure is designed to promote long productive steps for fast convergence. An analysis of the global convergence properties of the approach in the context of a line search Sequential Quadratic Programming method and numerical results for the KNITRO software package are presented.
\end{abstract}

Keywords: nonlinear programming, constrained optimization, sequential quadratic programming, penalty functions, global convergence

\section{Introduction}

In this paper we consider step acceptance mechanisms for nonlinear constrained optimization. For simplicity, we frame our discussion in the context of the equality constrained problem

$$
\begin{aligned}
& \min _{x \in \mathbb{R}^{n}} f(x) \\
& \text { s.t. } c(x)=0,
\end{aligned}
$$

where $f: \mathbb{R}^{n} \rightarrow \mathbb{R}$ and $c: \mathbb{R}^{n} \rightarrow \mathbb{R}^{t}$ are smooth functions, but consider ways in which our methods can be applied to problems with inequality constraints in the last section. The main purpose of this paper is to develop a globalization strategy designed to promote long productive steps and fast convergence, supported by convergence guarantees to first order optimal points.

Most globally convergent iterative algorithms for problem (1.1) have the following general form. At a given iterate $x_{k}$, a step is computed in either the primal or primal-dual space based on local and/or historical information of the problem functions. The step is then either accepted or rejected based on the reductions attained in the nonlinear objective $f(x)$, constraint infeasibility $\|c(x)\|$, or some combination of both measures. Here, $\|\cdot\|$ denotes a norm on $\mathbb{R}^{t}$. The manner in which these reductions are quantified and evaluated may have a significant impact on the types of steps accepted and the speed with which the algorithm converges to a solution.

We motivate our proposed globalization strategy, i.e., step acceptance method, by outlining two popular tools used for this purpose: exact penalty functions and filter mechanisms. The exact penalty

*This author was supported by Department of Energy grant DE-FG02-87ER25047-A004.

$\dagger$ This author was supported by National Science Foundation grant CCF-0514772 and a grant from the Intel Corporation. 
function we consider in this paper combines the objective and a constraint infeasibility measure into a function of the form

$$
\phi_{\pi}(x) \triangleq f(x)+\pi\|c(x)\|,
$$

where $\pi>0$ is a penalty parameter. During iteration $k$, a step is deemed acceptable only if a sufficient reduction in $\phi_{\pi_{k}}$ is attained for a suitable value of the penalty parameter. In contemporary algorithms, the value for $\pi_{k}$ is chosen upon completion of the step computation procedure and the sequence $\left\{\pi_{k}\right\}$ is typically monotonically increasing throughout the run of the algorithm. Figure 1 illustrates the region of acceptable points from $p_{k}=\left(\left\|c\left(x_{k}\right)\right\|, f\left(x_{k}\right)\right)$, corresponding to the current iterate $x_{k}$, in $\|c\|-f$ space. A step $d_{k}$ is acceptable if the resulting point $\bar{x}=x_{k}+d_{k}$ yields a pair $(\|c(\bar{x})\|, f(\bar{x}))$ lying sufficiently below the solid line through $p_{k}$, where the slope of the line is defined by the current value of the penalty parameter $\pi_{k}$. The global convergence properties of such an approach were first shown by Han (1977) and Powell (1978).

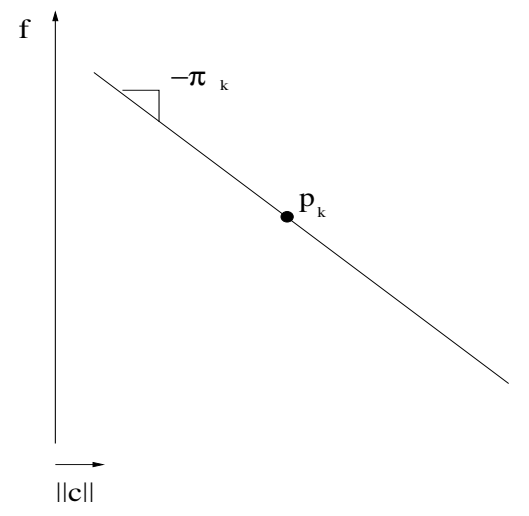

FIG. 1. Boundary of the region of acceptable points from $p_{k}$ for the penalty function $\phi_{\pi_{k}}$

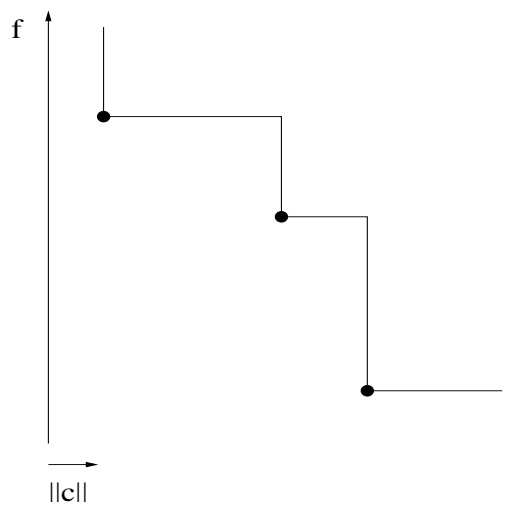

FIG. 2. Boundary of the region of acceptable points for a filter with three entries

A filter mechanism, proposed by Fletcher \& Leyffer (2002), avoids the definition of a parameter to balance reductions in the objective with reductions in the constraints. In the spirit of multiobjective optimization, a filter considers pairs of values $(\|c(x)\|, f(x))$ obtained by evaluating the functions $\|c\|$ and $f$ at all or some iterates preceding the current one. A pair $\left(\left\|c\left(x_{i}\right)\right\|, f\left(x_{i}\right)\right)$ is said to dominate another pair $\left(\left\|c\left(x_{j}\right)\right\|, f\left(x_{j}\right)\right)$ if and only if both $\left\|c\left(x_{i}\right)\right\| \leqslant\left\|c\left(x_{j}\right)\right\|$ and $f\left(x_{i}\right) \leqslant f\left(x_{j}\right)$. The filter $\mathscr{F}$ is then defined to be an index set corresponding to a list of pairs such that no pair dominates any other. A step $d_{k}$ from $x_{k}$ is considered acceptable if the resulting point $\bar{x}$ corresponds to a pair $(\|c(\bar{x})\|, f(\bar{x}))$ such that either

$$
\|c(\bar{x})\| \ll\left\|c\left(x_{i}\right)\right\| \text { or } f(\bar{x}) \ll f\left(x_{i}\right)
$$

for all $i \in \mathscr{F}$, where by "«" we mean that the value is less with respect to some appropriate margin. Upon the acceptance of such a step, the pair $(\|c(\bar{x})\|, f(\bar{x}))$ may be added to the filter, in which case all points dominated by this pair are removed from $\mathscr{F}$. Figure 2 illustrates the region of acceptable points for a filter with three entries as that lying sufficiently below and to the left of the piecewise linear function. The global convergence guarantees of such an approach have been shown when paired with certain types of step computation methods; e.g., see Fletcher et al. (1998), Gonzaga et al. (2003), and Wächter \& Biegler (2005a,b). 
Penalty functions and filter mechanisms both have their own advantages and disadvantages. One disadvantage of a penalty function relates to the monotonicity required when updating the penalty parameter $\pi$ during the solution process. Nonmonotone updates for the penalty parameter are available that maintain global convergence guarantees, but such methods often rely on ad hoc heuristics that eventually fall back on the convergence properties of monotone strategies, and so we do not discuss them here. Depending on the specific update strategy used, $\pi$ may at some point be set to an excessively large value, even at a point that is relatively far from a solution. As a result, a large priority will be placed on computing steps that produce sufficient reductions in constraint infeasibility, effectively "blocking" steps that move away from the feasible region. This can be detrimental as empirical evidence has shown that accepting steps that temporarily increase infeasibility can often lead to fast convergence. Figure 3 illustrates this blocking behavior of a penalty function, where we highlight the region of points that would be rejected despite the fact that each corresponding step would have provided a reduction in the objective $f$ (and so may have been acceptable to a filter).

We note that a second disadvantage of a penalty function is that a low value of $\pi$ may block steps that improve feasibility but increase $f$. However, modern step acceptance strategies effectively deal with this problem by defining local models of $\phi_{\pi}$ (as will be seen in Section 3), with which an adequately large value of $\pi$ can be determined to avoid excessive blocking. Thus, our view is that the main weakness of penalty-based strategies is the blocking effect illustrated in Figure 3, which can be particularly detrimental when $\left\|c_{k}\right\|$ is zero, or at least small, while $x_{k}$ is far from optimal.
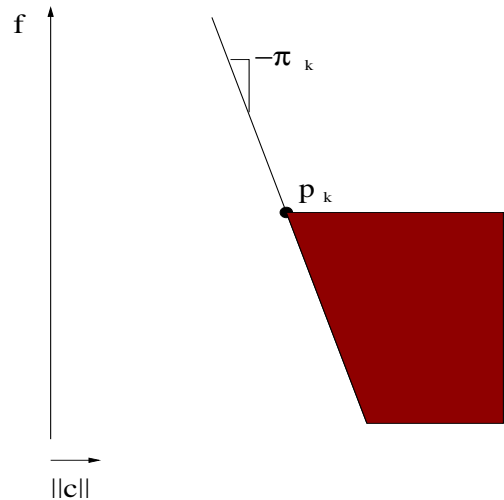

$\overrightarrow{\|c\|}$

FIG. 3. A region of points blocked by $\phi_{\pi_{k}}$

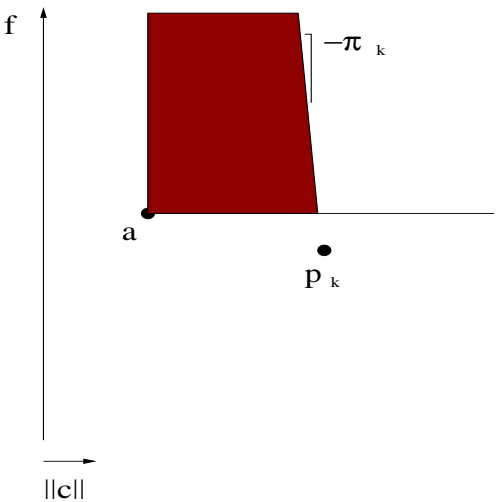

FIG. 4. A region of points blocked by a filter with entry $a$

One disadvantage of a filter mechanism is that a step can be blocked by a filter entry, i.e., historical information of the problem functions, when in fact the step is a productive move toward a solution in a local region of the search space. This is particularly worrisome when steps are blocked that would amount to a sufficient reduction in constraint infeasibility. Figure 4 depicts a filter with the single entry $a$ where the point $p_{k}=\left(\left\|c\left(x_{k}\right)\right\|, f\left(x_{k}\right)\right)$, corresponding to the current iterate $x_{k}$, is shown as the isolated point with an objective value sufficiently less than the filter entry. The shaded portion illustrates one region of points that are blocked by the filter, despite the fact that a step into this region would correspond to a reduction in constraint infeasibility from the current iterate (and so may be acceptable for a penalty function approach with parameter $\pi_{k}$ ).

In an extreme example, consider the case where the filter entry $a$ in Figure 4 is a Pareto optimal solution to the multiobjective optimization problem of minimizing the pair $(\|c(x)\|, f(x))$ over all $x \in \mathbb{R}^{n}$. A point is Pareto optimal if it cannot be dominated by any other point. Thus, if the current iterate again 
corresponds to the point $p_{k}$ in Figure 4, then all paths from $p_{k}$ to the feasible region must pass through a region of points dominated by $a$ in $\|c\|-f$ space. Feasibility can only be attained if a single computed step were to fall beyond the region dominated by the filter entry or if a backup mechanism, such as a feasibility restoration phase, were implemented.

In summary, both penalty functions and filters can be shown to block different types of productive steps. A penalty function may suffer from high priority being placed on improving feasibility and convergence can be slowed by forcing the algorithm to hug the feasible region. A filter mechanism, on the other hand, may suffer from handling problem (1.1) too much like a multiobjective optimization problem, when in fact a certain priority on converging to the feasible region may be appropriate, especially as the algorithm progresses.

\section{Flexible Penalty Functions}

In this section, we define a new step acceptance mechanism for nonlinear programming algorithms. By observing the strengths and weaknesses of penalty functions and filters, we hope to emulate some of the step acceptance behavior of both methods while attempting to avoid any blocking of productive steps.

During early iterations, the filter mechanism has the benefit that a variety of steps are considered acceptable. For example, for a one-element filter, i.e., a filter containing only an entry corresponding to the current iterate, a step will be accepted as long as a sufficient reduction in the objective or constraint infeasibility is attained. This may be of use to promote long steps during early iterations when an appropriate value for the penalty parameter may not yet be known. However, during later iterations, it may be reasonable to assume that an appropriate value for the penalty parameter may be determinable based on information computed throughout the run of the algorithm, which can be used to correctly block steps from increasing constraint infeasibility. The use of a penalty function in later iterations may also avoid the risk of blocking steps in the manner illustrated in Figure 4.

In an attempt to define a single mechanism that will capture all of these characteristics, and given that the penalty function approach appears to be more flexible than a filter in that it permits a reweighting of objective and constraint infeasibility measures, we present an improvement of the penalty strategies.

Our method can be motivated by observing the iterative nature of the penalty parameter update implemented in some current algorithms; e.g., see Waltz et al. (2006). At the start of iteration $k$, a specific value $\pi_{k-1}$ of the penalty parameter is carried over from the previous iteration. If the algorithm were to maintain this value, then only a step corresponding to a move into the region sufficiently below the solid line in Figure 5 would be acceptable. However, upon the calculation of $d_{k}$, the algorithm may determine that an increase of the penalty parameter to some value $\bar{\pi}_{k}>\pi_{k-1}$ may be appropriate, in which case only a step corresponding to a move into the region sufficiently below the dashed line in Figure 5 would be acceptable. Rather than automatically set $\pi_{k} \leftarrow \bar{\pi}_{k}$, a simple heuristic that maintains the global convergence properties of the algorithm is to first compute the function values for $\bar{x}=x_{k}+d_{k}$, namely $\|c(\bar{x})\|$ and $f(\bar{x})$. If $(\|c(\bar{x})\|, f(\bar{x}))$ lies sufficiently below the dashed line in Figure 5 , then we may accept the step and indeed set $\pi_{k} \leftarrow \bar{\pi}_{k}$. However, if $(\|c(\bar{x})\|, f(\bar{x}))$ lies sufficiently below the solid line, then the step could be considered acceptable for setting $\pi_{k} \leftarrow \pi_{k-1}$, effectively avoiding an increase in the penalty parameter. In summary, such a strategy does not consider a single value of $\pi$ at $x_{k}$, but rather may select from a pair of values depending on the actual reductions attained by the step. Thus, we can view the region of acceptable points as that lying below the solid or dashed line in Figure 5.

An extension of this idea forms the basis of the method we now propose. Consider the collection of 


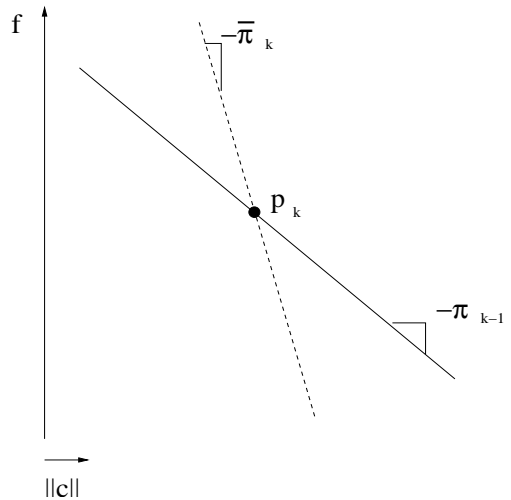

FIG. 5. Illustration of the iterative nature of penalty parameter updates

penalty functions

$$
\begin{aligned}
\phi_{\pi}(x) & \triangleq f(x)+\pi\|c(x)\|, \\
\pi & \in\left[\pi^{l}, \pi^{u}\right],
\end{aligned}
$$

for $0<\pi^{l} \leqslant \pi^{u}$. We define a step to be acceptable if a sufficient reduction in $\phi_{\pi}$ has been attained for at least one $\pi \in\left[\pi^{l}, \pi^{u}\right]$. Clearly, if $\pi^{l}$ is always chosen to equal $\pi^{u}$, then this approach is equivalent to using a penalty function with a fixed $\pi$ during each iteration. Alternatively, if $\pi^{l} \approx 0$ while $\pi^{u}$ is very large, then this approach has the form of a one-element filter. In general, the region of acceptable points is that given by the region down and to the left of the piecewise linear function illustrated in Figure 6, where the kink in the function always occurs at $p_{k}=\left(\left\|c\left(x_{k}\right)\right\|, f\left(x_{k}\right)\right)$, corresponding to the current iterate $x_{k}$. As the penalty parameter $\pi$ is allowed to fluctuate in the interval $\left[\pi^{l}, \pi^{u}\right]$, we refer to (2.1) as a "flexible" penalty function.

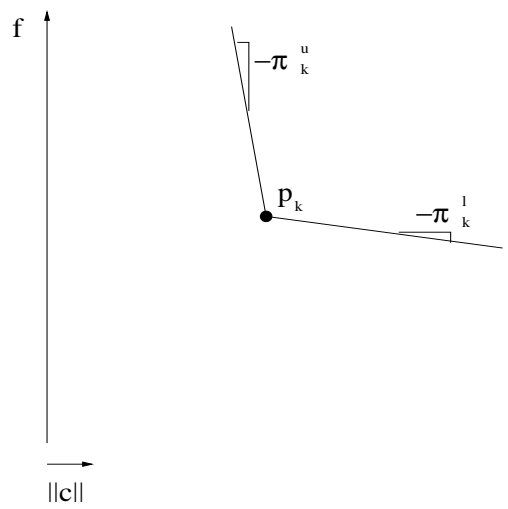

FIG. 6. Boundary of the region of acceptable points from $p_{k}$ for a flexible penalty function over $\left[\pi_{k}^{l}, \pi_{k}^{u}\right]$

Let us expound further on the relationship between our approach and some techniques that employ a filter by saying that the region of acceptable points in Figure 6 has features similar to the "slanting envelope" around a filter entry proposed by Chin \& Fletcher (2003) and considered later in a paper by 
$\mathrm{Li}$ (2006). However, despite the fact that the shape of the acceptable regions are similar in some areas of the $\|c\|-f$ plane, the important difference between our flexible penalty function and these and other filter mechanisms is that we do not maintain a collection of previous infeasibility measure/objective value pairs. The step acceptance criteria we propose for a flexible penalty function depend only on $\pi_{k}^{l}, \pi_{k}^{u}$, and constraint and objective information at the current iterate $x_{k}$.

The practical behavior of standard penalty function techniques depends heavily on the update strategy for the single parameter $\pi$. For a flexible penalty function, we need only consider the update strategies for two parameters: $\pi^{l}$ and $\pi^{u}$. As different requirements in terms of convergence guarantees are necessary for each of these boundary values, and as they have significantly different practical effects, we have the ability to design their updates in a manner suitable for accepting long productive steps.

We present a concrete strategy for updating $\pi^{l}$ and $\pi^{u}$ in the following section, as certain details are better described once features of the chosen step computation procedure are outlined.

NotATION: In the remainder of our discussion, we drop functional notation once values are clear from the context and delimit iteration number information for functions as with variables; i.e., we denote $f_{k} \triangleq f\left(x_{k}\right)$ and similarly for other quantities. We define $\|\cdot\|$ to be any fixed norm.

\section{A Line Search SQP Framework}

In this section we describe a precise globalization strategy for problem (1.1) based on the flexible penalty function (2.1) in the context of a line search Sequential Quadratic Programming (SQP) method.

Let us begin by formalizing a basic SQP method. The Lagrangian function for problem (1.1) is

$$
\mathscr{L}(x, \lambda) \triangleq f(x)+\lambda^{T} c(x),
$$

and the first-order optimality conditions are

$$
\nabla \mathscr{L}(x, \lambda)=\left[\begin{array}{c}
g(x)+A(x)^{T} \lambda \\
c(x)
\end{array}\right]=0,
$$

where $g(x) \triangleq \nabla f(x), A(x)$ is the Jacobian of $c(x)$, and $\lambda \in \mathbb{R}^{t}$ are Lagrange multipliers. The line search SQP methodology applied to problem (1.1) defines an appropriate displacement $d_{k}$ in the primal space from an iterate $x_{k}$ as the minimizer of a quadratic model of the objective subject to a linearization of the constraints. The quadratic program has the form

$$
\begin{aligned}
& \min _{d \in \mathbb{R}^{n}} f\left(x_{k}\right)+g\left(x_{k}\right)^{T} d+\frac{1}{2} d^{T} W\left(x_{k}, \lambda_{k}\right) d \\
& \text { s.t. } c\left(x_{k}\right)+A\left(x_{k}\right) d=0,
\end{aligned}
$$

where

$$
W(x, \lambda) \approx \nabla_{x x}^{2} \mathscr{L}(x, \lambda)=\nabla_{x x}^{2} f(x)+\sum_{i=1}^{t} \lambda^{i} \nabla_{x x}^{2} c^{i}(x)
$$

is equal to, or is a symmetric approximation for, the Hessian of the Lagrangian. Here, $c^{i}(x)$ and $\lambda^{i}$ denote the $i$ th constraint function and its corresponding dual variable, respectively. If the constraint Jacobian $A\left(x_{k}\right)$ has full row rank and $W\left(x_{k}, \lambda_{k}\right)$ is positive definite on the null space of $A\left(x_{k}\right)$, then a solution $d_{k}$ to (3.3) is well-defined and can be obtained via the solution of the linear system (see Nocedal \& Wright (2006)):

$$
\left[\begin{array}{cc}
W\left(x_{k}, \lambda_{k}\right) & A\left(x_{k}\right)^{T} \\
A\left(x_{k}\right) & 0
\end{array}\right]\left[\begin{array}{l}
d_{k} \\
\delta_{k}
\end{array}\right]=-\left[\begin{array}{c}
g\left(x_{k}\right)+A\left(x_{k}\right)^{T} \lambda_{k} \\
c\left(x_{k}\right)
\end{array}\right]
$$


The new iterate is then given by

$$
x_{k+1} \leftarrow x_{k}+\alpha_{k} d_{k},
$$

where the steplength coefficient $\alpha_{k} \in(0,1]$ is given by a globalization procedure. Here, we intend to employ the flexible penalty function (2.1), requiring appropriate update strategies for $\pi^{l}$ and $\pi^{u}$. In the following discussion, let us assume that $\left\|c_{k}\right\| \neq 0$ for each $k$. We comment on suitable updates for $\pi_{k}^{l}$ and $\pi_{k}^{u}$ in the special case of $\left\|c_{k}\right\|=0$ at the end of this section.

First, consider the parameter $\pi^{u}$. A large value of $\pi^{u}$ indicates that the algorithm considers almost any step that provides a sufficiently large reduction in constraint infeasibility to be acceptable. Thus, as approaching the feasible region is a necessity for any algorithm for solving problem (1.1), we may choose to initialize $\pi^{u}$ to a large value and increase it only when necessary. This can be done by updating $\pi^{u}$ in a manner currently used for setting $\pi$ in some contemporary penalty function approaches. The technique we have in mind makes decisions based on a model $m_{\pi}$ of the penalty function $\phi_{\pi}$, and in effect will increase $\pi$ (or, in our case, $\pi^{u}$ ) if and only if the computed step indicates that a large increase in the objective is likely to result from a reduction in constraint infeasibility.

Let us define a local model of $\phi_{\pi}$ around the current iterate $x_{k}$ as

$$
m_{\pi}(d)=f_{k}+g_{k}^{T} d+\frac{\omega(d)}{2} d^{T} W_{k} d+\pi\left\|c_{k}+A_{k} d\right\|,
$$

where

$$
\omega(d) \triangleq \begin{cases}1 & \text { if } d^{T} W_{k} d \geqslant 0 \\ 0 & \text { otherwise }\end{cases}
$$

(e.g., see El-Hallabi (1999), Byrd et al. (1999), Omojokun (1989), and Waltz et al. (2006)). Notice that $m_{\pi}$ contains a linear or quadratic model of the objective $f$ and a linear approximation of constraint infeasibility. With this approximation, we can estimate the reduction in $\phi_{\pi}$ attained by $d_{k}$ by evaluating

$$
\begin{aligned}
\operatorname{mred}_{\pi}\left(d_{k}\right) & \triangleq m_{\pi}(0)-m_{\pi}\left(d_{k}\right) \\
& =\left[-g_{k}^{T} d_{k}-\frac{\omega_{k}}{2} d_{k}^{T} W_{k} d_{k}\right]+\pi\left\|c_{k}\right\| .
\end{aligned}
$$

As the step $d_{k}$ satisfies the linearized constraints in problem (3.3), it follows that the model predicts no increase in constraint infeasibility, as evidenced by the nonnegative contribution of the last term in (3.6). Our model of the objective, however, may indicate that an increase or decrease in $f$ (corresponding to a negative or positive value, respectively, of the term in square brackets in (3.6)) is likely to occur along $d_{k}$. Overall, we consider the reduction in the model $m_{\pi}$ attained by $d_{k}$ to be sufficiently large if

$$
\operatorname{mred}_{\pi}\left(d_{k}\right) \geqslant \sigma \pi\left\|c_{k}\right\|
$$

for some $0<\sigma<1$, which can be seen to hold if

$$
\pi \geqslant \frac{g_{k}^{T} d_{k}+\frac{\omega_{k}}{2} d_{k}^{T} W_{k} d_{k}}{(1-\sigma)\left\|c_{k}\right\|} \triangleq \chi_{k} .
$$

Various algorithms will in fact enforce inequality (3.7), and so will set $\pi$ according to (3.8) for all $k$.

It turns out that our desired properties of $\pi^{u}$ can also be achieved by constructing an update around the term $\chi_{k}$. In particular, we propose a scheme of the form

$$
\pi_{k}^{u} \leftarrow \begin{cases}\pi_{k-1}^{u} & \text { if } \pi_{k-1}^{u} \geqslant \chi_{k} \\ \chi_{k}+\varepsilon & \text { otherwise, }\end{cases}
$$


where $\varepsilon>0$ is a small constant. In this manner, $\pi^{u}$ will be increased during an iteration if and only if an increase in the model objective, reflected by a positive numerator in (3.8), indicates that an increase in $f$ is likely to occur in conjunction with a move toward the feasible region, implied by the fact that the step satisfies the linearized constraints in (3.3).

By using the model $m_{\pi}$ to set a value for the penalty parameter, the resulting sequence of values can be shown to remain bounded under common assumptions due to certain desirable properties of the quadratic subproblem (3.3). (This phenomenon, which remains important for our flexible penalty function approach in the context of $\pi^{u}$, can be observed more precisely in our proof of Lemma 3.6 in the following section.) A drawback of this technique, however, is that such a model may not always accurately reflect changes in the objective and constraint values. For example, mred $_{\pi}$ may suggest that a move along $d_{k}$ corresponds to a decrease in constraint infeasibility and an increase in the objective, when in fact the opposite may occur if one were to take the full step $d_{k}$. As such, the penalty parameter may be set to a large value that results in excessive blocking in later iterations. Further motivation for incorporating a flexible penalty function, therefore, results from the fact that an excessively large value for $\pi_{k}^{u}$ is less of a concern if the penalty parameter is able to fluctuate over an interval $\left[\pi_{k}^{l}, \pi_{k}^{u}\right]$ during the line search - especially if the mechanism for choosing $\pi_{k}^{l}$ is not based on local models of the functions at all.

The method we propose for setting $\pi^{l}$ is such a technique. In particular, we choose to have $\pi_{k}^{l}$ set in a manner that reflects the actual reductions in $f$ and $\|c\|$ attained during the previous iteration $k-1$ (where $\pi_{0}^{l}$ is provided as a small initial value).

To motivate the details of the scheme we propose, consider the numbered regions illustrated in Figure 7, where the position and shape of each portion depends on the parameters $\pi_{k}^{l}$ (set during iteration $k-1)$ and $\pi_{k}^{u}$, and the location of the point $p_{k}=\left(\left\|c\left(x_{k}\right)\right\|, f\left(x_{k}\right)\right)$. A step into region I would not be acceptable to the flexible penalty function (2.1), as opposed to a step into region II, III, or IV, which would be acceptable. Our strategy for setting $\pi_{k+1}^{l}$ will depend on the region in $\|c\|-f$ space to which

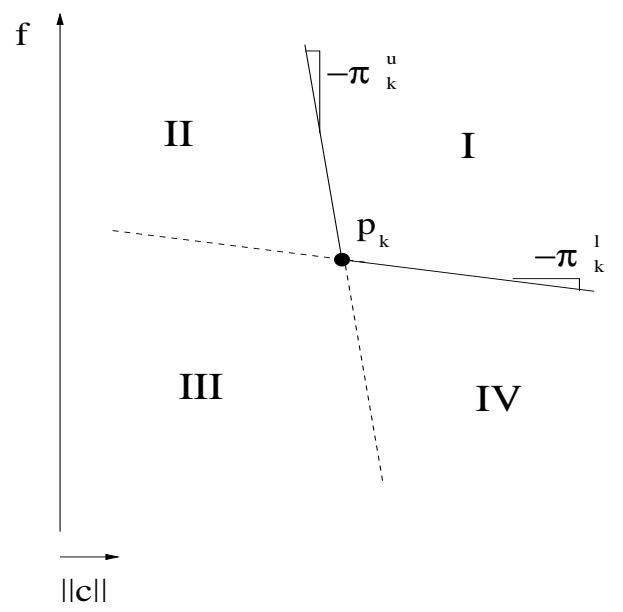

FIG. 7. Distinct regions defined by the current state of a flexible penalty function

the step $\alpha_{k} d_{k}$ moved upon the conclusion of the line search. If a sufficient reduction in $\phi_{\pi_{k}^{l}}$ was obtained (i.e., the step was into region III or IV), then we say that the reductions in $f$ and/or $\|c\|$ are sufficient for the current state of the flexible penalty function and so we set $\pi_{k+1}^{l} \leftarrow \pi_{k}^{l}$. Otherwise, (i.e., if the 
step was into region II), $\pi^{l}$ will be increased. This has the logical interpretation that we only become more restrictive by blocking steps that increase infeasibility when the algorithm is confronted with steps that indicate that actual moves toward the feasible region correspond to actual increases in the objective (thus freeing ourselves from being bound by parameters set based on models or other local information). The precise update after a step into region II is given by

$$
\pi_{k+1}^{l} \leftarrow \min \left\{\pi_{k}^{u}, \pi_{k}^{l}+\max \left\{0.1\left(v-\pi_{k}^{l}\right), \varepsilon^{l}\right\}\right\}
$$

where $\varepsilon^{l}>0$ is some small constant and

$$
v=\frac{f\left(x_{k}+\alpha_{k} d_{k}\right)-f\left(x_{k}\right)}{\left\|c\left(x_{k}\right)\right\|-\left\|c\left(x_{k}+\alpha_{k} d_{k}\right)\right\|} .
$$

Here, the definition of $v$ ensures that the value for $\pi_{k+1}^{l}$ depends on the actual reductions in the objective and constraint infeasibility attained by $\alpha_{k} d_{k}$, where it can be seen that $v \in\left[\pi_{k}^{l}, \pi_{k}^{u}\right]$ after a step into region II. We introduce the damping factor 0.1 so that the value for $\pi^{l}$ will increase only gradually, thus blocking as few future steps as possible while still ensuring convergence.

Our procedures for updating the state of the flexible penalty function (2.1) are now set. Before presenting the algorithm in detail, however, let us remark on an important detail of the line search procedure for computing $\alpha_{k}$. With $D \phi_{\pi}\left(d_{k}\right)$ denoted as the directional derivative of $\phi_{\pi}$ along $d_{k}$, we require that $\alpha_{k}$ satisfy the Armijo condition

$$
\begin{aligned}
& \phi_{\pi}\left(x_{k}+\alpha_{k} d_{k}\right) \leqslant \phi_{\pi}\left(x_{k}\right)+\eta \alpha_{k} D \phi_{\pi_{k}^{m}}\left(d_{k}\right), \\
& \quad \text { for some } \pi \in\left[\pi_{k}^{l}, \pi_{k}^{u}\right],
\end{aligned}
$$

where $0<\eta<1$ and $\pi_{k}^{m} \in\left[\pi_{k}^{l}, \pi_{k}^{u}\right]$. Note that we have defined a parameter $\pi_{k}^{m}$ for calculating a single value of the directional derivative, which must be chosen to ensure that this term is sufficiently negative for each $k$. This could be achieved by choosing $\pi_{k}^{m}=\pi_{k}^{u}$ for all $k$ (see Lemma 3.5). However, as seen in Theorem 18.2 of Nocedal \& Wright (2006), the directional derivative is given by

$$
D \phi_{\pi_{k}^{m}}\left(d_{k}\right)=g_{k}^{T} d_{k}-\pi_{k}^{m}\left\|c_{k}\right\|
$$

and so larger values of $\pi_{k}^{m}$ will make this term more negative. As fewer values of $\alpha_{k}$ will satisfy (3.12) for more negative values of $D \phi_{\pi_{k}^{m}}\left(d_{k}\right)$, we would like to choose $\pi_{k}^{m}$ in the interval $\left[\pi_{k}^{l}, \pi_{k}^{u}\right]$ so that this term is negative enough to ensure sufficient descent, while also being as close to zero as possible so as to allow the largest number of acceptable steplengths. We use

$$
\pi_{k}^{m} \leftarrow \max \left\{\pi_{k}^{l}, \chi_{k}\right\}
$$

which, along with (3.9) and the fact that $\pi_{k}^{l} \leqslant \pi_{k-1}^{u}$ (see (3.10)), ensures $\pi_{k}^{m} \geqslant \chi_{k}$ and $\pi_{k}^{m} \in\left[\pi_{k}^{l}, \pi_{k}^{u}\right]$.

Overall, we have described the following algorithm.

\section{Algorithm 3.1 Line Search SQP Method with a Flexible Penalty Function}

Initialize $x_{0}, \lambda_{0}, 0<\pi_{0}^{l} \leqslant \pi_{-1}^{u}, 0<\varepsilon, \varepsilon^{l}$, and $0<\eta, \sigma<1$

for $k=0,1,2, \ldots$, until a convergence test for problem (1.1) is satisfied

Compute $f_{k}, g_{k}, c_{k}, W_{k}$, and $A_{k}$ and set $\alpha_{k} \leftarrow 1$

Compute $\left(d_{k}, \delta_{k}\right)$ via (3.4) 
If $c_{k} \neq 0$, set $\pi_{k}^{u}$ according to (3.9) and $\pi_{k}^{m}$ by (3.14); else, set $\pi_{k}^{u} \leftarrow \pi_{k-1}^{u}$ and $\pi_{k}^{m} \leftarrow \pi_{k-1}^{m}$ until the Armijo condition (3.12) holds for some $\pi \in\left[\pi_{k}^{l}, \pi_{k}^{u}\right]$, set $\alpha_{k} \leftarrow \alpha_{k} / 2$

If the Armijo condition (3.12) holds for $\pi=\pi_{k}^{l}$, set $\pi_{k+1}^{l} \leftarrow \pi_{k}^{l}$; else, set $\pi_{k+1}^{l}$ by (3.10) $\operatorname{Set}\left(x_{k+1}, \lambda_{k+1}\right) \leftarrow\left(x_{k}, \lambda_{k}\right)+\alpha_{k}\left(d_{k}, \delta_{k}\right)$ endfor

A practical implementation of the line search procedure of Algorithm 3.1 is attained by the observation that, during iteration $k$, the Armijo condition (3.12) is satisfied for $\pi \in\left[\pi_{k}^{l}, \pi_{k}^{u}\right]$ if and only if it is satisfied for either $\pi=\pi_{k}^{l}$ or $\pi=\pi_{k}^{u}$. Thus, the line search for a given step $d_{k}$ can be performed simply by evaluating the reductions attained in $\phi_{\pi_{k}^{l}}$ and $\phi_{\pi_{k}^{u}}$. We also note that in the special case of $\left\|c_{k}\right\|=0$ during iteration $k$, we maintain $\pi_{k}^{u} \leftarrow \pi_{k-1}^{u}$ as in this case the directional derivative $D \phi_{\pi}\left(d_{k}\right)$ is independent of $\pi$ (see (3.13)). We can also trivially set $\pi_{k}^{m} \leftarrow \pi_{k}^{l}$ and maintain $\pi_{k+1}^{l} \leftarrow \pi_{k}^{l}$ since in this setting region II of Figure 7 is empty.

\subsection{Global Analysis}

In this section we explore the global convergence properties of Algorithm 3.1 under the following assumptions.

Assumptions 3.1 The sequence $\left\{x_{k}, \lambda_{k}\right\}$ generated by Algorithm 3.1 is contained in a convex set $\Omega$ and the following properties hold:

(a) The functions $f$ and $c$ and their first and second derivatives are bounded on $\Omega$.

(b) The constraint Jacobians $A_{k}$ have full row rank and their smallest singular values are bounded below by a positive constant.

(c) The sequence $\left\{W_{k}\right\}$ is bounded.

(d) There exists a constant $\mu>0$ such that over all $k$ and for any $u \in \mathbb{R}^{n}$ with $u \neq 0$ and $A_{k} u=0$ we have $u^{T} W_{k} u \geqslant \mu\|u\|^{2}$.

These assumptions are fairly standard for a line search method; e.g., see Han (1977) and Powell (1983). Assumption 3.1(b), however, is strong, but we use it to simplify the analysis in order to focus on the issues related to the incorporation of a flexible penalty function. Assuming that $W_{k}$ is positive definite on the null space of the constraints is natural for line search algorithms, for otherwise there would be no guarantee of descent.

Our analysis hinges on our ability to show that the algorithm will eventually compute an infinite sequence of steps that sufficiently reduce the penalty function $\phi_{\pi^{l}}$ for a fixed $\pi^{l}>0$, which we achieve by following the approach taken in Byrd et al. (2007) for an inexact SQP method. In particular, we consider the decomposition

$$
d_{k}=u_{k}+v_{k}
$$

where the tangential component $u_{k}$ lies in the null space of the constraint Jacobian $A_{k}$ and the normal component $v_{k}$ lies in the range space of $A_{k}^{T}$. The components are not to be computed explicitly; the decomposition is only for analytical purposes. We refer to $u_{k}$, which by definition satisfies $A_{k} u_{k}=0$, as the tangential component and $v_{k}$ as the normal component.

We first present a result related to the length of the primal step $d_{k}$ and the sequence of Lagrange multiplier estimates $\left\{\lambda_{k}\right\}$. 
LEMMA 3.1 For all $k$, the primal step $d_{k}$ is bounded in norm. Moreover, the sequence of Lagrange multipliers $\left\{\lambda_{k}\right\}$ is bounded.

Proof. Under Assumptions 3.1, it can be shown that the primal-dual matrix in (3.4) is nonsingular and that its inverse is bounded in norm over all $k$ (e.g., see Nocedal \& Wright (2006)). Thus, the relation

$$
\left[\begin{array}{c}
d_{k} \\
\lambda_{k}+\delta_{k}
\end{array}\right]=-\left[\begin{array}{cc}
W_{k} & A_{k}^{T} \\
A_{k} & 0
\end{array}\right]^{-1}\left[\begin{array}{l}
g_{k} \\
c_{k}
\end{array}\right]
$$

implies that $\left\|\left(d_{k}, \lambda_{k}+\delta_{k}\right)\right\| \leqslant \gamma\left\|\left(g_{k}, c_{k}\right)\right\|$ holds over all $k$ for some constant $\gamma>0$. The results then follow from $\alpha_{k} \leqslant 1$ and the fact that Assumption 3.1(a) implies that $\left\|\left(g_{k}, c_{k}\right)\right\|$ is bounded over all $k$.

The next result ensures a precise bound on the length of the normal component $v_{k}$ with respect to the current value of the infeasibility measure.

LEMMA 3.2 There exists $\gamma_{1}>0$ such that, for all $k$,

$$
\left\|v_{k}\right\|^{2} \leqslant \gamma_{1}\left\|c_{k}\right\|
$$

Proof. From $A_{k} v_{k}=A_{k} d_{k}=-c_{k}$ and the fact that $v_{k}$ lies in the range space of $A_{k}^{T}$, it follows that

$$
v_{k}=-A_{k}^{T}\left(A_{k} A_{k}^{T}\right)^{-1} c_{k},
$$

and so

$$
\left\|v_{k}\right\| \leqslant\left\|A_{k}^{T}\left(A_{k} A_{k}^{T}\right)^{-1}\right\|\left\|c_{k}\right\| .
$$

The result follows from the facts that Assumption 3.1(a) states that $\left\|c_{k}\right\|$ is bounded and Assumptions 3.1(a) and (b) imply that $\left\|A_{k}^{T}\left(A_{k} A_{k}^{T}\right)^{-1}\right\|$ is bounded.

We now turn to the following result concerning an important property of the tangential steps.

LEMmA 3.3 There exists a constant $\gamma_{2}>0$ such that, over all $k$, if $\left\|u_{k}\right\|^{2} \geqslant \gamma_{2}\left\|v_{k}\right\|^{2}$ then $\frac{1}{2} d_{k}^{T} W_{k} d_{k} \geqslant$ $\frac{\mu}{4}\left\|u_{k}\right\|^{2}$.

Proof. Assumption 3.1(d) implies that for any $\gamma_{2}>0$ such that $\left\|u_{k}\right\|^{2} \geqslant \gamma_{2}\left\|v_{k}\right\|^{2}$ we have

$$
\begin{aligned}
\frac{1}{2} d_{k}^{T} W_{k} d_{k} & =\frac{1}{2} u_{k}^{T} W_{k} u_{k}+u_{k}^{T} W_{k} v_{k}+\frac{1}{2} v_{k}^{T} W_{k} u_{k} \\
& \geqslant \frac{\mu}{2}\left\|u_{k}\right\|^{2}-\left\|u_{k}\right\|\left\|W_{k}\right\|\left\|v_{k}\right\|-\frac{1}{2}\left\|W_{k}\right\|\left\|v_{k}\right\|^{2} \\
& \geqslant\left(\frac{\mu}{2}-\frac{\left\|W_{k}\right\|}{\sqrt{\gamma_{2}}}-\frac{\left\|W_{k}\right\|}{2 \gamma_{2}}\right)\left\|u_{k}\right\|^{2} .
\end{aligned}
$$

Thus, with Assumption 3.1(c) we have that the result holds for some sufficiently large $\gamma_{2}>0$.

With the above results, we can now identify two types of iterations. Let $\gamma_{2}>0$ be chosen large enough as described in Lemma 3.3 and consider the sets of indices

$$
\begin{array}{r}
K_{1} \triangleq\left\{k:\left\|u_{k}\right\|^{2} \geqslant \gamma_{2}\left\|v_{k}\right\|^{2}\right\} \\
\text { and } K_{2} \triangleq\left\{k:\left\|u_{k}\right\|^{2}<\gamma_{2}\left\|v_{k}\right\|^{2}\right\} .
\end{array}
$$

Our remaining analysis will be dependent on these sets and the corresponding quantity

$$
\Theta_{k} \triangleq \begin{cases}\left\|u_{k}\right\|^{2}+\left\|c_{k}\right\|, & k \in K_{1} \\ \left\|c_{k}\right\|, & k \in K_{2}\end{cases}
$$

The quantity $\Theta_{k}$ will help us form a common bound for the length of the primal step and the quantity $D \phi_{\pi_{k}^{m}}\left(d_{k}\right)$. 
LEMma 3.4 There exists $\gamma_{3}>1$ such that, for all $k$,

$$
\left\|d_{k}\right\|^{2} \leqslant \gamma_{3} \Theta_{k}
$$

and hence,

$$
\left\|d_{k}\right\|^{2}+\left\|c_{k}\right\| \leqslant 2 \gamma_{3} \Theta_{k}
$$

Proof. For $k \in K_{1}$, Lemma 3.2 implies

$$
\left\|d_{k}\right\|^{2}=\left\|u_{k}\right\|^{2}+\left\|v_{k}\right\|^{2} \leqslant\left\|u_{k}\right\|^{2}+\gamma_{1}\left\|c_{k}\right\| .
$$

Similarly, Lemma 3.2 implies that for $k \in K_{2}$

$$
\left\|d_{k}\right\|^{2}=\left\|u_{k}\right\|^{2}+\left\|v_{k}\right\|^{2}<\left(\gamma_{2}+1\right)\left\|v_{k}\right\|^{2} \leqslant\left(\gamma_{2}+1\right) \gamma_{1}\left\|c_{k}\right\| .
$$

To establish (3.17) we note that $\Theta_{k}+\left\|c_{k}\right\| \leqslant 2 \Theta_{k}$ for all $k$.

The next result bounds the quantity $D \phi_{\pi_{k}^{m}}\left(d_{k}\right)$, where $\pi_{k}^{m}$ is defined by (3.14).

LEMMA 3.5 There exists $\gamma_{4}>0$ such that, for all $k$,

$$
D \phi_{\pi_{k}^{m}}\left(d_{k}\right) \leqslant-\gamma_{4} \Theta_{k}
$$

Proof. Recall that by Theorem 18.2 in Nocedal \& Wright (2006) we have

$$
D \phi_{\pi_{k}^{m}}\left(d_{k}\right)=g_{k}^{T} d_{k}-\pi_{k}^{m}\left\|c_{k}\right\|
$$

If $\left\|c_{k}\right\|=0$, then (3.4) yields

$$
D \phi_{\pi_{k}^{m}}\left(d_{k}\right)=g_{k}^{T} d_{k}=-d_{k}^{T} W_{k} d_{k} .
$$

Lemmas 3.2 and 3.3 then imply $\left\|v_{k}\right\|=0$ and $k \in K_{1}$, and so

$$
D \phi_{\pi_{k}^{m}}\left(d_{k}\right)=-d_{k}^{T} W_{k} d_{k} \leqslant-\frac{\mu}{2}\left\|u_{k}\right\|^{2}
$$

and the result holds for $\gamma_{4}=\frac{\mu}{2}$.

Now suppose $\left\|c_{k}\right\| \neq 0$. Here, (3.8), (3.18), and the fact that (3.14) implies $\pi_{k}^{m} \geqslant \chi_{k}$, yields

$$
D \phi_{\pi_{k}^{m}}\left(d_{k}\right) \leqslant-\frac{\omega_{k}}{2} d_{k}^{T} W_{k} d_{k}-\sigma \pi_{k}^{m}\left\|c_{k}\right\| .
$$

By Lemma 3.3 and (3.5), we have that $\omega_{k}=1$ for $k \in K_{1}$ and thus

$$
D \phi_{\pi_{k}^{m}}\left(d_{k}\right) \leqslant-\frac{\mu}{4}\left\|u_{k}\right\|^{2}-\sigma \pi_{k}^{m}\left\|c_{k}\right\|
$$

Similarly, for $k \in K_{2}$ we have from (3.5) and (3.19) that

$$
D \phi_{\pi_{k}^{m}}\left(d_{k}\right) \leqslant-\sigma \pi_{k}^{m}\left\|c_{k}\right\|
$$

The result holds for $\gamma_{4}=\min \left\{\frac{\mu}{4}, \sigma \pi_{k}^{m}\right\}$, which is positive as $\pi_{k}^{m} \geqslant \pi_{k}^{l} \geqslant \pi_{0}^{l}>0$ for all $k$.

An important property of Algorithm 3.1 is that under Assumptions 3.1 the sequence $\left\{\pi_{k}^{u}\right\}$ remains bounded. We prove this result next.

LEMMA 3.6 The sequence $\left\{\pi_{k}^{u}\right\}$ is bounded above and $\pi_{k}^{u}$ remains constant for all sufficiently large $k$. 
Proof. Recall that $\pi_{k}^{u}$ is set during iteration $k$ of Algorithm 3.1 to satisfy (3.8), which is equivalent to saying that (3.7) will be satisfied, as in

$$
-g_{k}^{T} d_{k}-\frac{\omega_{k}}{2} d_{k}^{T} W_{k} d_{k}+(1-\sigma) \pi_{k}^{u}\left\|c_{k}\right\| \geqslant 0 .
$$

If $d_{k}^{T} W_{k} d_{k} \geqslant 0$, then $\omega_{k}=1$ by (3.5) and so (3.4) and Lemma 3.1 imply that there exists $\gamma_{5}>0$ such that

$$
-g_{k}^{T} d_{k}-\frac{1}{2} d_{k}^{T} W_{k} d_{k}=\frac{1}{2} d_{k}^{T} W_{k} d_{k}-c_{k}^{T}\left(\lambda_{k}+\delta_{k}\right) \geqslant-\gamma_{5}\left\|c_{k}\right\|
$$

Similarly, if $d_{k}^{T} W_{k} d_{k}<0$, then $\omega_{k}=0, k \in K_{2}$, and $\left\|d_{k}\right\|^{2} \leqslant \gamma_{3}\left\|c_{k}\right\|$ by Lemma 3.4. Then, Assumptions 3.1, (3.4), and Lemma 3.1 imply that there exists $\gamma_{6}, \gamma_{6}^{\prime}>0$ such that

$$
-g_{k}^{T} d_{k}-\frac{\omega_{k}}{2} d_{k}^{T} W_{k} d_{k}=d_{k}^{T} W_{k} d_{k}-c_{k}^{T}\left(\lambda_{k}+\delta_{k}\right) \geqslant-\gamma_{6}\left(\left\|d_{k}\right\|^{2}+\left\|c_{k}\right\|\right) \geqslant-\gamma_{6}\left\|c_{k}\right\| .
$$

These results together imply that for all $k$,

$$
-g_{k}^{T} d_{k}-\frac{\omega_{k}}{2} d_{k}^{T} W_{k} d_{k} \geqslant-\max \left\{\gamma_{5}, \gamma_{6}\right\}\left\|c_{k}\right\|,
$$

and so (3.20), and equivalently (3.7), is always satisfied if

$$
\pi_{k}^{u} \geqslant \max \left\{\gamma_{5}, \gamma_{6}^{\prime}\right\} /(1-\sigma)
$$

Therefore, if $\pi_{\bar{k}}^{u} \geqslant \max \left\{\gamma_{5}, \gamma_{6}^{\prime}\right\} /(1-\sigma)$ for some iteration number $\bar{k} \geqslant 0$, then $\pi_{k}^{u}=\pi_{\bar{k}}^{u}$ for all $k \geqslant \bar{k}$. This, together with the fact that whenever Algorithm 3.1 increases $\pi^{u}$ it does so by at least a positive finite amount, proves the result.

A similar result can be shown for the parameter $\pi^{l}$.

COROLLARY $3.1\left\{\pi_{k}^{l}\right\}$ is bounded above and $\pi_{k}^{l}$ remains constant for all sufficiently large $k$.

Proof. By Lemma 3.6, $\pi_{k}^{u}$ is constant for all sufficiently large $k$. Then, we have by (3.10) that if $\pi^{l}$ is increased, then it is done so by at least a finite constant amount, or it is set equal to $\pi^{u}$. Thus, the result follows from (3.10) and the fact that there can only be a finite number of increases of $\pi^{l}$.

The previous lemmas can be used to bound the sequence of steplength coefficients.

LEMMA 3.7 The sequence $\left\{\alpha_{k}\right\}$ is bounded below by a positive constant.

Proof. Let us rewrite the Armijo condition (3.12) for convenience as

$$
\phi_{\pi}\left(x_{k}+\alpha_{k} d_{k}\right)-\phi_{\pi}\left(x_{k}\right) \leqslant \eta \alpha_{k} D \phi_{\pi_{k}^{m}}\left(d_{k}\right)
$$

for $\pi \in\left[\pi_{k}^{l}, \pi_{k}^{u}\right]$. Suppose that the line search fails for some $\bar{\alpha}>0$, which means that (3.21) does not hold for any $\pi \in\left[\pi_{k}^{l}, \pi_{k}^{u}\right]$. In particular,

$$
\phi_{\pi_{k}^{m}}\left(x_{k}+\bar{\alpha} d_{k}\right)-\phi_{\pi_{k}^{m}}\left(x_{k}\right)>\eta \bar{\alpha} D \phi_{\pi_{k}^{m}}\left(d_{k}\right),
$$

where we recall that $\pi_{k}^{m} \in\left[\pi_{k}^{l}, \pi_{k}^{u}\right]$. As seen on page 541 of Nocedal \& Wright (2006), it can be shown under Assumptions 3.1 that for some $\gamma_{7}>0$ we have

$$
\phi_{\pi_{k}^{m}}\left(x_{k}+\bar{\alpha} d_{k}\right)-\phi_{\pi_{k}^{m}}\left(x_{k}\right) \leqslant \bar{\alpha} D \phi_{\pi_{k}^{m}}\left(d_{k}\right)+\bar{\alpha}^{2} \gamma_{7} \pi_{k}^{m}\left\|d_{k}\right\|^{2},
$$

so

$$
(\eta-1) D \phi_{\pi_{k}^{m}}\left(d_{k}\right)<\bar{\alpha} \gamma_{\uparrow} \pi_{k}^{m}\left\|d_{k}\right\|^{2} .
$$


Lemmas 3.4 and 3.5 then yield

$$
(1-\eta) \gamma_{4} \Theta_{k}<\bar{\alpha} \gamma_{3} \gamma_{7} \pi_{k}^{m} \Theta_{k}
$$

so

$$
\bar{\alpha}>(1-\eta) \gamma_{4} /\left(\gamma_{3} \gamma_{7} \pi_{k}^{m}\right)>(1-\eta) \gamma_{4} /\left(\gamma_{3} \gamma_{7} \pi_{k}^{u}\right) .
$$

Thus, $\alpha_{k}$ is never set below $(1-\eta) \gamma_{4} /\left(2 \gamma_{3} \gamma_{7} \pi_{k}^{u}\right)$, which is bounded below and away from zero by Lemma 3.6, in order to satisfy the Armijo condition (3.12) for some $\pi \in\left[\pi_{k}^{l}, \pi_{k}^{u}\right]$.

We are now ready to present the main result of this section.

THEOREM 3.2 Algorithm 3.1 yields

$$
\lim _{k \rightarrow \infty}\left\|\left[\begin{array}{c}
g_{k}+A_{k}^{T} \lambda_{k} \\
c_{k}
\end{array}\right]\right\|=0 .
$$

Proof. By Corollary 3.1 the algorithm eventually computes, during a certain iteration $k^{*} \geqslant 0$, a finite value $\pi^{*}$ beyond which the value of the parameter $\pi^{l}$ will never be increased. This means that for all sufficiently large $k$, the Armijo condition (3.12) is satisfied for $\pi^{l}=\pi^{*}$, or else $\pi^{l}$ would be increased (see the second-to-last line of Algorithm 3.1). From Lemmas 3.5 and 3.7, we then have that for all $k \geqslant k^{*}$

$$
\phi_{\pi^{*}}\left(x_{k}\right)-\phi_{\pi^{*}}\left(x_{k}+\alpha_{k} d_{k}\right) \geqslant \gamma_{8} \Theta_{k}
$$

for some $\gamma_{8}>0$. Therefore, (3.17) implies

$$
\begin{aligned}
\phi_{\pi^{*}}\left(x_{k^{*}}\right)-\phi_{\pi^{*}}\left(x_{k}\right) & =\sum_{j=k^{*}}^{k-1}\left(\phi_{\pi^{*}}\left(x_{j}\right)-\phi_{\pi^{*}}\left(x_{j+1}\right)\right) \\
& \geqslant \gamma_{8} \sum_{j=k^{*}}^{k-1} \Theta_{j} \\
& \geqslant \frac{\gamma_{8}}{2 \gamma_{3}} \sum_{j=k^{*}}^{k-1}\left(\left\|d_{j}\right\|^{2}+\left\|c_{j}\right\|\right),
\end{aligned}
$$

and so

$$
\lim _{k \rightarrow \infty}\left\|d_{k}\right\|=0 \quad \text { and } \quad \lim _{k \rightarrow \infty}\left\|c_{k}\right\|=0
$$

follow from the fact that Assumption 3.1(a) implies $\phi_{\pi^{*}}$ is bounded below. Finally, the first block equation of (3.4), Assumption 3.1(c), and Lemma 3.1 imply

$$
\begin{aligned}
\left\|g_{k+1}+A_{k+1}^{T} \lambda_{k+1}\right\| & =\left\|g_{k}+A_{k}^{T} \lambda_{k+1}+\left(g_{k+1}-g_{k}\right)+\left(A_{k+1}-A_{k}\right)^{T} \lambda_{k+1}\right\| \\
& =\left\|\left(1-\alpha_{k}\right)\left(g_{k}+A_{k}^{T} \lambda_{k}\right)-\alpha_{k} W_{k} d_{k}+\left(g_{k+1}-g_{k}\right)+\left(A_{k+1}-A_{k}\right)^{T} \lambda_{k+1}\right\| \\
& \leqslant\left(1-\alpha_{k}\right)\left\|g_{k}+A_{k}^{T} \lambda_{k}\right\|+O\left(\left\|d_{k}\right\|\right)
\end{aligned}
$$

and so

$$
\lim _{k \rightarrow \infty}\left\|g_{k}+A_{k}^{T} \lambda_{k}\right\|=0
$$

follows from (3.22), the fact that $\alpha_{k} \leqslant 1$, and Lemma 3.7. 


\section{Numerical Results}

In this section we present numerical results for a particular implementation of Algorithm 3.1 incorporated into the KNITRO-Direct algorithm in the KNITRO 5.0 software package; see Waltz \& Plantenga (2006) for details. We tested the code using a set of 85 equality constrained problems from the CUTEr (see Bongartz et al. (1995) and Gould et al. (2003)) and COPS (see Dolan et al. (2004)) collections. From these sets, we chose problems for which AMPL models were readily available. The default KNITRO-Direct algorithm may revert to a trust region iteration to handle negative curvature and to ensure global convergence. In our tests, we enabled internal options to prevent this from occurring. Instead, the algorithm modifies $W_{k}$ if necessary to ensure that the resulting matrix is positive definite on the null space of $A_{k}$ - to ensure that our implementation performs as a pure line search algorithm.

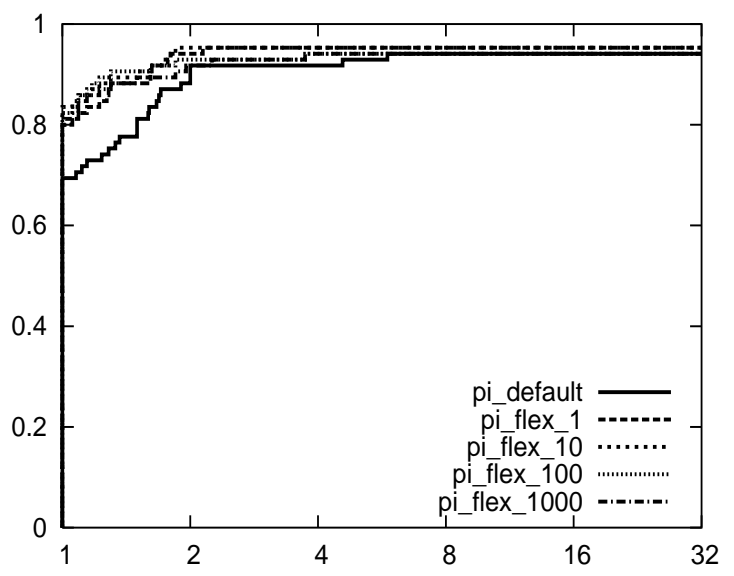

FIG. 8. Performance profile for iterations

As the globalization strategy described in this paper incurs little computational cost and is designed to promote long steps for fast convergence, we propose that the numbers of iterations and function evaluations required to find a solution are appropriate measures for comparison with other methods. We compare the results of an algorithm using the default penalty function approach in KNITRO-Direct, call it pi_default, with the results using a flexible penalty function. The penalty parameter update strategy in KNITRO-Direct corresponds to the case when (3.10) is replaced by $\pi_{k+1}^{l} \leftarrow \pi_{k}^{u}$. For pi_default and the algorithm with a flexible penalty function, we initialize $\pi$ and $\pi^{l}$ to $10^{-8}$, respectively. We consider the four initial values $1,10,100$, and 1000 for $\pi^{u}$, which correspond to the algorithms we refer to as pi_flex_1, pi_flex_10, pi_flex_100, and pi_flex_1000, respectively. Table 1 contains a complete listing of the input parameters for our implementation of Algorithm 3.1.

The results for the five algorithms are summarized in Figures 8 and 9 in terms of logarithmic performance profiles, as described in Dolan \& Moré (2002). Here, the leftmost values indicate the proportion of times each algorithm solves a given problem using the least value of the given measure; i.e., number of iterations or of function evaluations. The values fail to add to one as ties are present. The rightmost function values illustrate the robustness of each approach; i.e., the percentage of times that a given problem is solved. 


\begin{tabular}{|l|r|}
\hline Parameter & Value \\
\hline$\pi_{0}^{l}$ & $10^{-8}$ \\
$\pi_{-1}^{u}$ & $\{1,10,100,1000\}$ \\
$\varepsilon$ & $10^{-4}$ \\
$\varepsilon^{l}$ & $10^{-4}$ \\
$\eta$ & $10^{-8}$ \\
$\sigma$ & $10^{-1}$ \\
\hline
\end{tabular}

Table 1. Input values for Algorithm 3.1

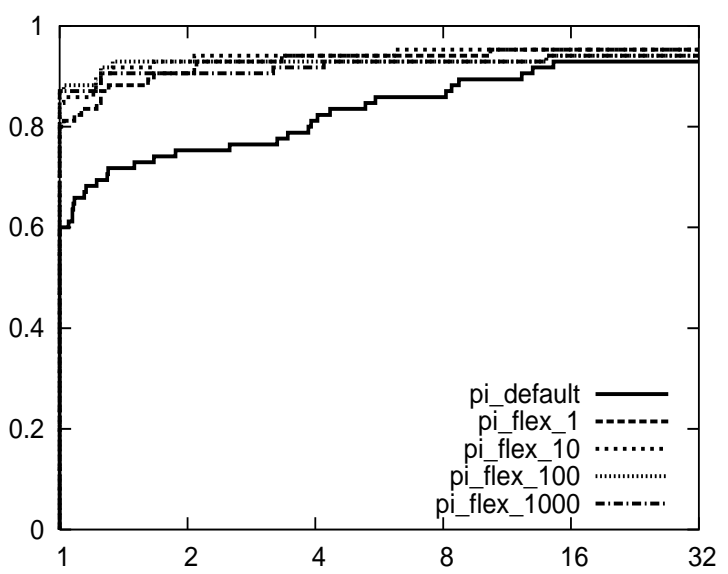

FIG. 9. Performance profile for function evaluations

The results are encouraging. Not only does an algorithm with a flexible penalty function approach often require slightly fewer iterations to find a solution, but a considerable amount of savings is often experienced in terms of function evaluations. This can be understood as the line search procedure generally has to perform fewer backtracks for a given step, leading to longer steps and a higher percentage of unit steplengths (i.e., full Newton steps). We also observe that the plots for pi_flex_1, pi_flex_10, pi_flex_100, and pi_flex_1000 are nearly indistinguishable throughout much of Figures 8 and 9. This suggests that the initial value for $\pi^{u}$ is inconsequential compared to the effect that separate updating strategies for $\pi^{l}$ and $\pi^{u}$ has on the practical performance of the approach.

\section{Final Remarks}

In this paper we have proposed and analyzed a new globalization strategy for equality constrained optimization problems. Our flexible penalty function allows for relatively unrestricted movement during early iterations, but also automatically tightens itself to forcefully guide convergence when necessary, thus manipulating the search appropriately throughout a run of the algorithm. An example of a particular implementation of the mechanism was presented in the context of a line search SQP method, after which the global behavior was analyzed and successful numerical results were outlined.

We close by describing how the ideas of this paper might be extended to generally constrained 
problems of the form

$$
\begin{array}{rl}
\min _{x \in \mathbb{R}^{n}} & f(x) \\
\text { s.t. } & c^{E}(x)=0, \\
& c^{I}(x) \leqslant 0,
\end{array}
$$

where $f: \mathbb{R}^{n} \rightarrow \mathbb{R}, c^{E}: \mathbb{R}^{n} \rightarrow \mathbb{R}^{t^{E}}$, and $c^{I}: \mathbb{R}^{n} \rightarrow \mathbb{R}^{t^{I}}$ are smooth functions. One of the leading classes of methods for solving problem (5.1) are interior-point approaches. Some algorithms of this type begin by introducing a log-barrier term with parameter $\mu>0$ for the inequalities into the objective to form the perturbed problem

$$
\begin{gathered}
\min _{x \in \mathbb{R}^{n}} f(x)-\mu \sum_{i \in I} \ln s^{i} \\
\text { s.t. } c^{E}(x)=0, \\
c^{I}(x)+s=0 .
\end{gathered}
$$

A solution for problem (5.1) is then found via the (approximate) solution of a sequence of problems of the form (5.2) for $\mu \rightarrow 0$, where throughout the process the vector of slack variables $s=\left(s^{1}, \ldots, s^{t^{I}}\right) \in \mathbb{R}^{t^{I}}$ is forced to be positive. Thus, for each given $\mu>0$ we can define the flexible penalty function associated with the barrier subproblem (5.2) as

$$
\begin{aligned}
\varphi_{\pi}(x) & \triangleq f(x)-\mu \sum_{i \in I} \ln s^{i}+\pi\left\|\left[\begin{array}{c}
c^{E}(x) \\
c^{I}(x)+s
\end{array}\right]\right\|, \\
\pi & \in\left[\pi^{l}, \pi^{u}\right]
\end{aligned}
$$

where $0 \leqslant \pi^{l} \leqslant \pi^{u}$, and a line search algorithm similar to Algorithm 3.1 can be applied. (The discussion here refers to a generic algorithm; to obtain practical methods with global convergence guarantees, various safeguards or modifications must be added. One such modification is the penalty function regularization described in Chen \& Goldfarb (2006).)

A similar approach can be used in a trust region algorithm. Here, a step $d_{k}$ from $x_{k}$ is typically accepted if and only if the actual reduction in a penalty function $\phi_{\pi}$, defined by

$$
\operatorname{pred}_{\pi}\left(d_{k}\right) \triangleq \phi_{\pi}\left(x_{k}\right)-\phi_{\pi}\left(x_{k}+d_{k}\right),
$$

is large with respect to the reduction obtained in a model such as $m_{\pi}$ (see Section 3 ). This condition can be written as

$$
\frac{\operatorname{pred}_{\pi}\left(d_{k}\right)}{\operatorname{mred}_{\pi}\left(d_{k}\right)} \geqslant \eta
$$

for some $0<\eta<1$, where it should be observed that we may now have $\left\|c_{k}+A_{k} d_{k}\right\|>0$. Rather than restrict the step acceptance criteria to this inequality with a fixed $\pi>0$ during each iteration $k$, we claim that an effect similar to that expressed in this paper can be achieved if instead a step is considered acceptable if

$$
\frac{\operatorname{pred}_{\pi_{k}^{l}}\left(d_{k}\right)}{\operatorname{mred}_{\pi_{k}^{m}}\left(d_{k}\right)} \geqslant \eta \text { or } \frac{\operatorname{pred}_{\pi_{k}^{u}}\left(d_{k}\right)}{\operatorname{mred}_{\pi_{k}^{m}}\left(d_{k}\right)} \geqslant \eta,
$$

where $\left[\pi_{k}^{l}, \pi_{k}^{u}\right]$ is a prescribed interval and $\pi_{k}^{m} \in\left[\pi_{k}^{l}, \pi_{k}^{u}\right]$ is chosen carefully so that $\operatorname{mred}_{\pi_{k}^{m}}\left(d_{k}\right)$ is sufficiently positive. All of the quantities $\pi_{k}^{l}$, $\pi_{k}^{u}$, and $\pi_{k}^{m}$ can be defined and updated in a manner similar to that described in this paper. 
The previous discussion outlines ways in which our flexible penalty function can be employed in the context of constrained optimization. We note, however, that in order to obtain practical algorithms with global convergence guarantees, various algorithmic components must be added to the methods described above.

ACKNOWLEDGMENTS: The authors are grateful to Richard Byrd and Richard Waltz for a number of productive discussions on this work. They also acknowledge a useful discussion with Sven Leyffer, Philippe Toint, and Andreas Wächter about filter mechanisms and penalty functions.

\section{REFERENCES}

Bongartz, I., Conn, A. R., Gould, N. I. M. \& Toint, Ph. L. (1995) CUTE: Constrained and Unconstrained Testing Environment, ACM Transactions on Mathematical Software, 21(1), 123-160.

BYrd, R. H., CURTIS, F. E., \& NocedAl, J. (2007) Inexact SQP Methods for Equality Constrained Optimization, to appear in SIAM Journal on Optimization.

Byrd, R. H., Gilbert, J.-Ch., \& Nocedal, J. (2000) A trust region method based on interior point techniques for nonlinear programming, Mathematical Programming, 89, 149-185.

Byrd, R. H., Hribar, M. E. \& Nocedal, J. (1999) An interior point algorithm for large scale nonlinear programming, SIAM Journal on Optimization, 9(4), 877-900.

BYRD, R. H. \& NocedAl, J. (1994) An analysis of reduced Hessian methods for constrained optimization, Mathematical Programming, 63, 129-156.

Chen, L. \& Goldfarb, D. (2006) Interior-point $l_{2}$ Penalty Methods for Nonlinear Programming with Strong Global Convergence Properties, Mathematical Programming, 108(1), 1-36.

Chin, C. M. \& Fletcher, R. (2003) On the global convergence of an SLP-filter algorithm that takes EQP steps, Mathematical Programming, 91(1), 161-177.

Dolan, E. D. \& Moré, J. J. (2002) Benchmarking optimization software with performance profiles, Mathematical Programming, Series A, 91, 201-213.

Dolan, E. D., Moré, J. J. \& Munson, T. S. (2004) Benchmarking optimization software with COPS 3.0, Technical Report ANL/MCS-TM-273, Argonne National Laboratory, Argonne, Illinois, USA.

EL-HALLABI, M. (1999) A hybrid algorithm for nonlinear equality constrained optimization problems: global and local convergence theory, Technical Report TR4-99, Mathematics and Computer Science Department, Institut National des Postes et Télécommunications, Rabat, Morocco.

Fletcher, R. \& LeyfFer, S. (2002) Nonlinear programming without a penalty function, Mathematical Programming, Series A, 91, 239-269.

Fletcher, R., LeyfFer, S. \& Toint, Ph. L. (1998) On the global convergence of an SLP-filter algorithm, Technical Report 98/13, Department of Mathematics, University of Namur, Namur, Belgium.

Gonzaga, C. C., KARAS, E. \& VANTI, M. (2003) A globally convergent filter method for nonlinear programming, SIAM Journal on Optimization, 14(3), 646-669.

Gould, N. I. M., Orban, D. \& Toint, Ph. L. (2003) CUTEr and sifdec: A Constrained and Unconstrained Testing Environment, revisited, ACM Trans. Math. Softw., 29(4), 373-394.

HAN, S. P. (1977) A globally convergent method for nonlinear programming, Journal of Optimization Theory and Applications, 22(3), 297-309.

LI, D. Q. (2006) A new SQP-filter method for solving nonlinear programming problems, Journal of Computational Mathematics, 24(5), 609-634.

Nocedal, J. \& Wright, S. J. (2006) Numerical Optimization, Second Edition, Springer Series in Operations Research, New York: Springer.

OMOJOKUn, E. O. (1989) Trust region algorithms for optimization with nonlinear equality and inequality constraints, PhD thesis, University of Colorado, Boulder, Colorado, USA. 
Powell, M. J. D. (1978) A fast algorithm for nonlinearly constrained optimization calculations, In: WATSON, G. A., ed., Numerical Analysis, Dundee 1977, number 630 in Lecture Notes in Mathematics, Heidelberg, Berlin, New York: Springer-Verlag, 144-157.

Powell, M. J. D. (1983) Variable metric methods for constrained optimization, In: BACHEM, A., GRÖTSCHEL, M. \& Korte, B., eds. Mathematical Programming: The State of the Art, Bonn: Springer-Verlag, 288-311.

W ÄCHTER, A. \& BIEGLER, L. T. (2005b) Line search filter methods for nonlinear programming: Local convergence, SIAM Journal on Optimization, 16(1), 32-48.

WÄCHTER, A. \& BIEGLER, L. T. (2005a) Line search filter methods for nonlinear programming: Motivation and global convergence, SIAM Journal on Optimization, 16(1), 1-31.

Waltz, R. A., Morales, J. L., Nocedal, J. \& Orban, D. (2006) An interior algorithm for nonlinear optimization that combines line search and trust region steps, Mathematical Programming, Series A, 107, 391-408.

Waltz, R. A. \& Plantenga, T. (2006) KNITRO User's Manual, Ziena Optimization, Inc., Evanston, IL, USA. 